\title{
National statistics for diet, alcohol consumption, and chronic pancreatitis in England and Wales, 1960-88
}

\author{
C D Johnson, S Hosking
}

\begin{abstract}
This study compared national statistics for consumption of alcohol and dietary intake with the numbers of cases of chronic pancreatitis as recorded in a $10 \%$ sample of all hospital discharges (Hospital In-Patient Enquiry) and with the annual number of deaths. Hospital discharges for chronic pancreatitis became more common throughout the study period (7.0-11.1 discharges per million per year in $1960-4 ; 26 \cdot 8-32.4$ discharges per million per year in 1980-4). The most rapid increase was from 1975-82. The rate increased fourfold in men, but only twofold in women, and the increase began earlier in men (1976) than in women (1980). Annual deaths from chronic pancreatitis also rose progressively (46-70 in $1960-4 ; 64-91$ in 1985-8). The annual per capita consumption of alcohol rose from 4.0 4.9 litres in 1960-4 to a peak of 7.7 litres in 1979; it has been relatively stable $(6.9-7.6$ litres) in 1980-8. Total dietary energy intake assessed in the National Food Survey fell progressively, with a correspondingly greater fall in carbohydrate intake, so that the energy contributions of fat and protein rose slightly, although total fat and protein consumption fell from 1965-9 to 1980-4. These changes were small in relation to total dietary intake and seemed to be unrelated to the changes in the numbers of chronic pancreatitis discharges. There was a close correlation $(r=0.96)$ between per capita alcohol consumption and the numbers of discharges with chronic pancreatitis six years later. This suggests that epidemiological trends in chronic pancreatitis in the United Kingdom might be predictable from population based statistics of alcohol consumption.
\end{abstract}

The causal relation of alcohol consumption to chronic pancreatitis is well established ${ }^{12}$ but the relation between duration of consumption and onset of symptoms, and between changes in national trends for consumption and disease statistics are far from clear. Another factor implicated in this disease is diet, but its precise role also varies in different reports. ${ }^{13}$ Most studies have drawn their conclusions from the investigation of a selected group of patients after they have presented with clinical evidence of chronic pancreatitis. The inevitable lag time between any dietary change and the development of chronic pancreatitis makes it difficult to determine with accuracy details of a patient's diet for the preceding five to 10 years. Furthermore, the disease itself may modify the diet.

For these reasons we have examined published national statistics in England and Wales span- ning a 29 year period. In this survey we have examined changes in diet and alcohol consumption, as well as the changing prevalence and mortality of chronic pancreatitis to ascertain their interrelations during this time period. These data may serve as an indication of future trends for this disease.

\section{Methods}

We studied the numbers of hospital discharges for chronic pancreatitis and dietary composition from 1960 to 1984 and the quantity of alcohol consumed from 1960 to 1988 . The numbers of discharges for chronic pancreatitis was determined from the Hospital In-Patient Enquiry of the Office of Population Censuses and Surveys. The inquiry recorded the diagnosis of a $10 \%$ sample of all hospital discharges from 1962-3 to 1984-5. The inquiry stopped after 1984 and its replacement, Korner, has yet to publish details of inpatient activity. An estimate of the 'population rate' of chronic pancreatitis discharges from $1962-3$ to $1984-5$ was made using the figures from the inquiry, with the appropriate multiplication factor and population figures published by the Office of Population Censuses and Surveys. We studied national figures as well as data from three regions (North West Thames, South West, and Yorkshire). Annual numbers of deaths from chronic pancreatitis were determined for 1960-88 from statistics published by the Office of Population Censuses and Surveys.

Dietary composition was determined from the National Food Survey of the Ministry of Agriculture, Fisheries and Food. This survey records all food consumed during a period of one week in 1000 households. The households studied are selected from all areas of the United Kingdom and are stratified by social class. The study takes place throughout the year to reduce seasonal bias. The National Food Survey does not include food consumed outside the home, confectionery, or alcohol. The survey produces national figures, as well as data for regions corresponding to the health service regions. Total caloric intake and the relative contribution to the diet and total intake of fat, protein, and carbohydrate are all recorded. Figures for consumption of sugar, chocolate, and confectionery were obtained from the appropriate trade organisation. ${ }^{4}$

National figures for alcohol consumption were obtained from the published data for alcohol production and importation derived from duty paid to HM Customs and Excise. ${ }^{5}$ We have no information for regional variations in alcohol consumption.

The relation between dietary changes, alterations in alcohol consumption, and variations in
University Surgical Southampton General Hospital, Tremona Road, C D Johnson

$S$ Hosking

Accepted for publication

28 January 1991 
Annual hospital discharges per million population, deaths from chronic pancreatitis, and per capital alcohol, chocolate, and sugar consumption in five year periods 1960-88. Data expressed as median (range). Disease figures for England and Wales. Alcohol, sugar, and chocolate consumption for United Kingdom

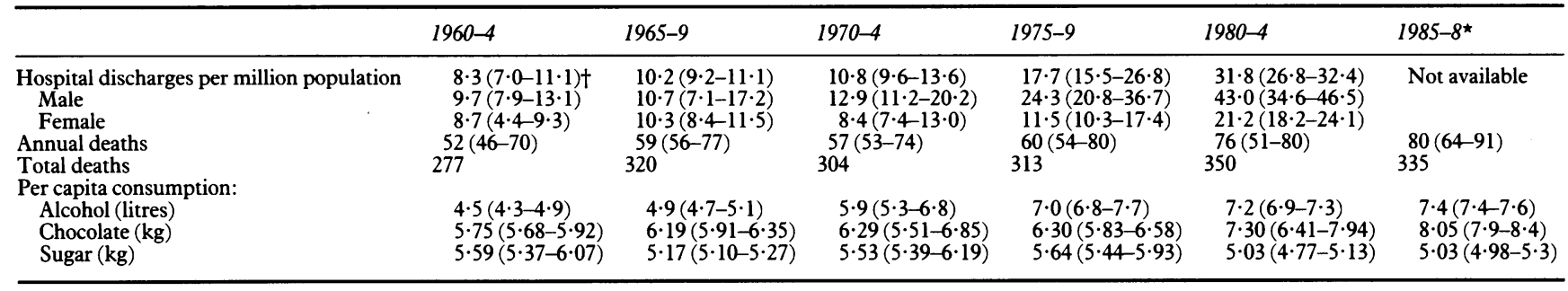

^Data for four years only; tdata for three years only.

the discharge rate of chronic pancreatitis were examined by graphic expression of the data. Least square regression analysis was done on selected data. The apparent relation between alcohol consumption and chronic pancreatitis was tested by linear regression. The validity of the least squares analysis depends on the independence of the residuals from the model. Since the data are ordered in time it is possible that this may not be the case. The DurbinWatson statistic ${ }^{6}$ which tests for serial correlation, was computed to examine the independence of the residuals. Rates of discharges and deaths were grouped into five year periods (1960-4 et seq) and expressed as median and range. These figures were compared using the Kruskal-Wallis analysis of variance.

\section{Results}

The rate of hospital discharges for chronic pancreatitis rose slowly during the years 1962-74 from 7 to 13.6 discharges per million population per annum. The rate then rose more rapidly but seems to have levelled off in 1980-4 (Table and Fig 1). It is of interest that the trend was different in men and women. Discharges per million in men rose about fourfold beginning in the mid 1970 s, whereas the figures for women show about a twofold increase beginning in 1979 .

The median number of deaths per annum from chronic pancreatitis in each five year period between 1960 and 1979 was fairly constant, at 50-60 per annum (Table and Fig 1). After 1979 the number of deaths increased $(p<0.05$, Kruskal-Wallis analysis of variance) to 64-91 per annum in 1985-8.

Estimated total daily energy intake recorded by the National Food Survey fell progressively during the study period, from $2640 \mathrm{kcal}$ to 2060

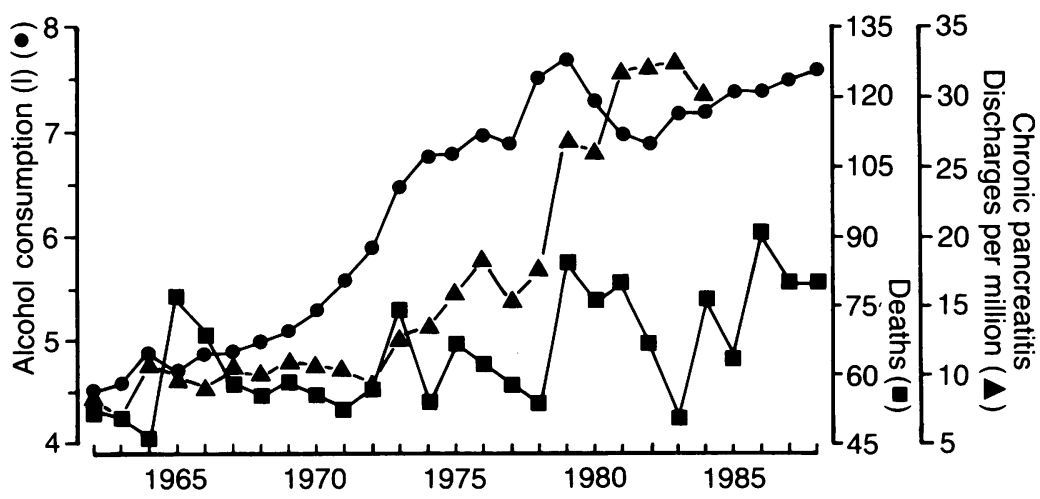

Figure 1: Changes in annual alcohol consumption (litres per capita), hospital discharges for chronic pancreatitis per million population, and total deaths from chronic pancreatitis. $\mathrm{kcal}(11 \cdot 0 \mathrm{MJ}$ to $9 \cdot 1 \mathrm{MJ})$. This corresponded to falls in total intake of all the major dietary components (Fig 2). From 1960-4 to 1980-4 mean daily fat consumption fell from $116 \mathrm{~g}$ to $102 \mathrm{~g}$, mean daily protein consumption from $75.3 \mathrm{~g}$ to $69.9 \mathrm{~g}$ and mean daily carbohydrate consumption from $341 \mathrm{~g}$ to $257 \mathrm{~g}$. Fat and protein consumption were similar in the first two quinquennia, and fell progressively thereafter. The fall in carbohydrate consumption was progressive throughout the period under review.

The fall in carbohydrate consumption was proportionately greater than that for fat or protein, with the result that the percentage of recorded energy intake as carbohydrate fell from $48.7 \%$ in $1960-4$ to $44.5 \%$ in $1980-4$ (Fig 2A). The estimated percentage contribution of both fat and protein increased slightly. Consumption of chocolate increased slowly during the first 20 years of the study, and more rapidly in 1980-4 (Table). Total sugar consumption was variable, lacking an obvious trend. From 1975-85, per capita sugar based confectionery consumption fell from $5.48 \mathrm{~kg}$ in 1975 to $5.13 \mathrm{~kg}$ in 1980 and $5.03 \mathrm{~kg}$ in 1985 . Corresponding figures for chocolate confectionery showed a rise from 5.83 $\mathrm{kg}$ in 1975 to $6.41 \mathrm{~kg}$ in 1980 , and $8.11 \mathrm{~kg}$ in 1985.

The changes with time in dietary composition recorded by the National Food Survey were evenly distributed across the 25 years of the study, whereas confectionery consumption was variable, but rose rapidly after 1980 . Both seemed to be unrelated to changes in the number of patients discharged from hospital with the diagnosis of chronic pancreatitis. There were only small regional differences in dietary intake and composition. These differences were less than the changes observed nationally during the study period. (Data not shown.) There was no discernible regional difference in the rate of hospital discharges with chronic pancreatitis.

Alcohol consumption increased during the period under study. The most rapid rise was from 1970-80 (Fig 1). After falling in the early 1980 s, total consumption increased by $10 \%$ per year over the next six years, almost reaching the high level recorded in 1979 . In $1960,73 \%$ of all alcohol consumed was contained in beer; in 1984 this figure had fallen to $58 \%$. In contrast, the equivalent figures for wine rose from $8 \%$ in 1960 to $18 \%$ in 1984 .

The curves for alcohol consumption and discharges of patients with chronic pancreatitis shown in Figure 1 seem to be approximately parallel, with the increase in chronic pancreatitis following the rise in alcohol consumption by five 
(A)

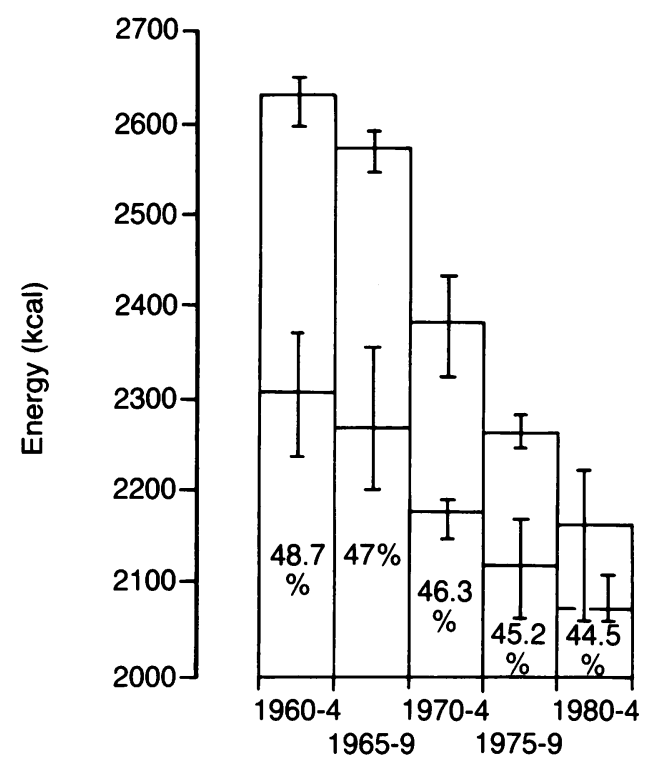

(C)

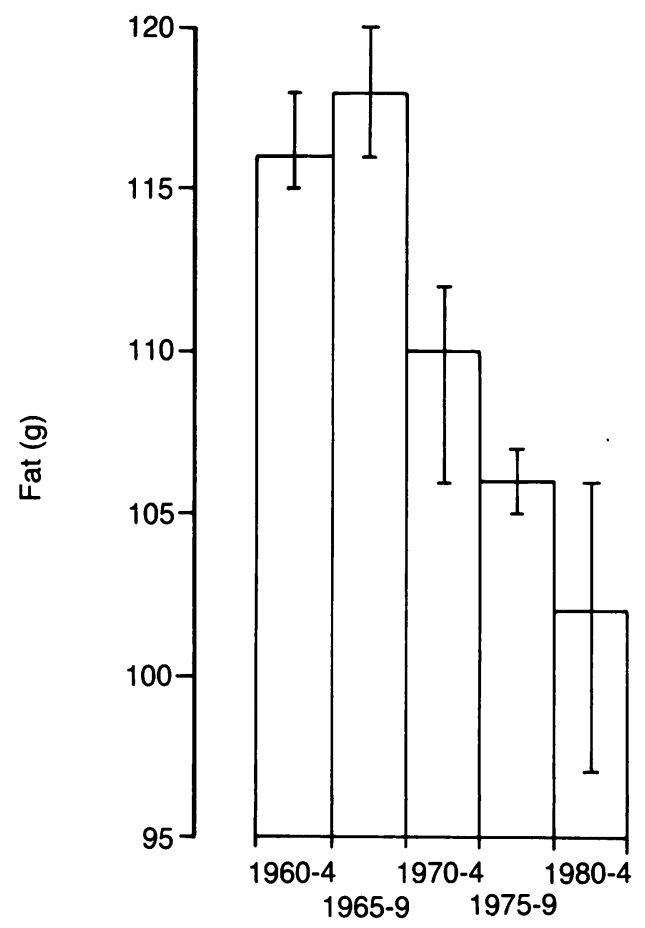

to seven years. Regression analysis of annual alcohol consumption with the numbers of chronic pancreatitis discharges at each yearly interval from four to eight years later showed that the best correlation between alcohol consumption and discharges of chronic pancreatitis patients occurred six years apart $(y=8 x-26 \cdot 4$, $r=0.96 ; n=20$, when $x=$ annual alcohol consumption (litres) and $\mathrm{y}=$ chronic pancreatitis (discharges per million), Fig 3). These data were computed to give the Durbin-Watson statistic $(d)=1.58$. The critical range for $d$ is 1.13 to 1.38 , and as both $\mathrm{d}$ and $4-\mathrm{d}$ lie outside this range, serial correlation of the residuals is not signifi-
(B)

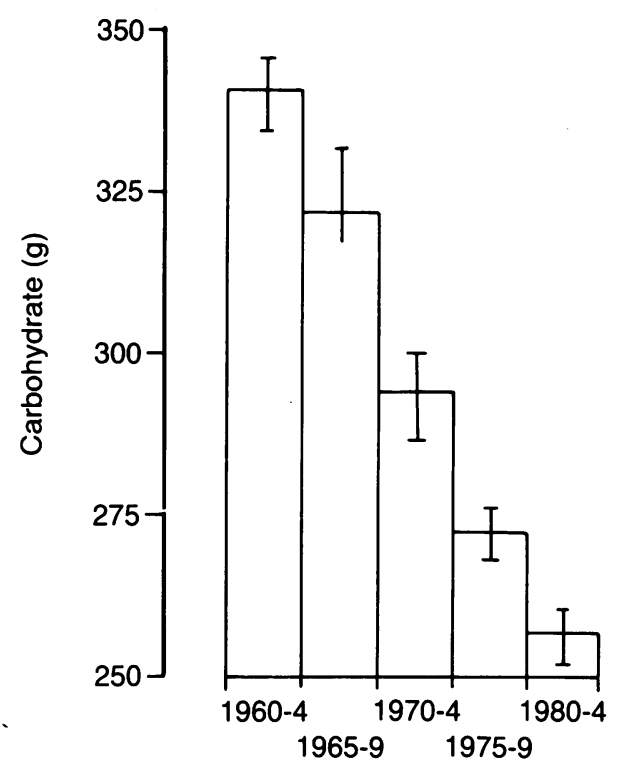

(D)

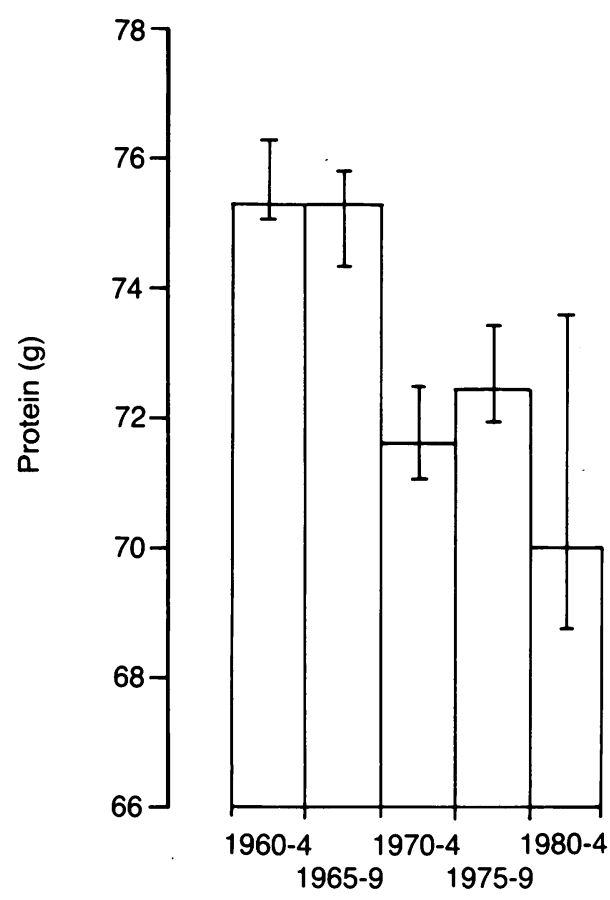

cant, which implies that the correlation observed with the regression analysis is genuine.

\section{Discussion}

This is the first study of chronic pancreatitis in the United Kingdom based on national statistics. The data presented confirm the clinical impression that chronic pancreatitis has increased in frequency and that this is closely related to changes in alcohol consumption.

These data are subject to the shortcomings inherent in nationally collected statistics. It is impossible to define diagnostic criteria for 


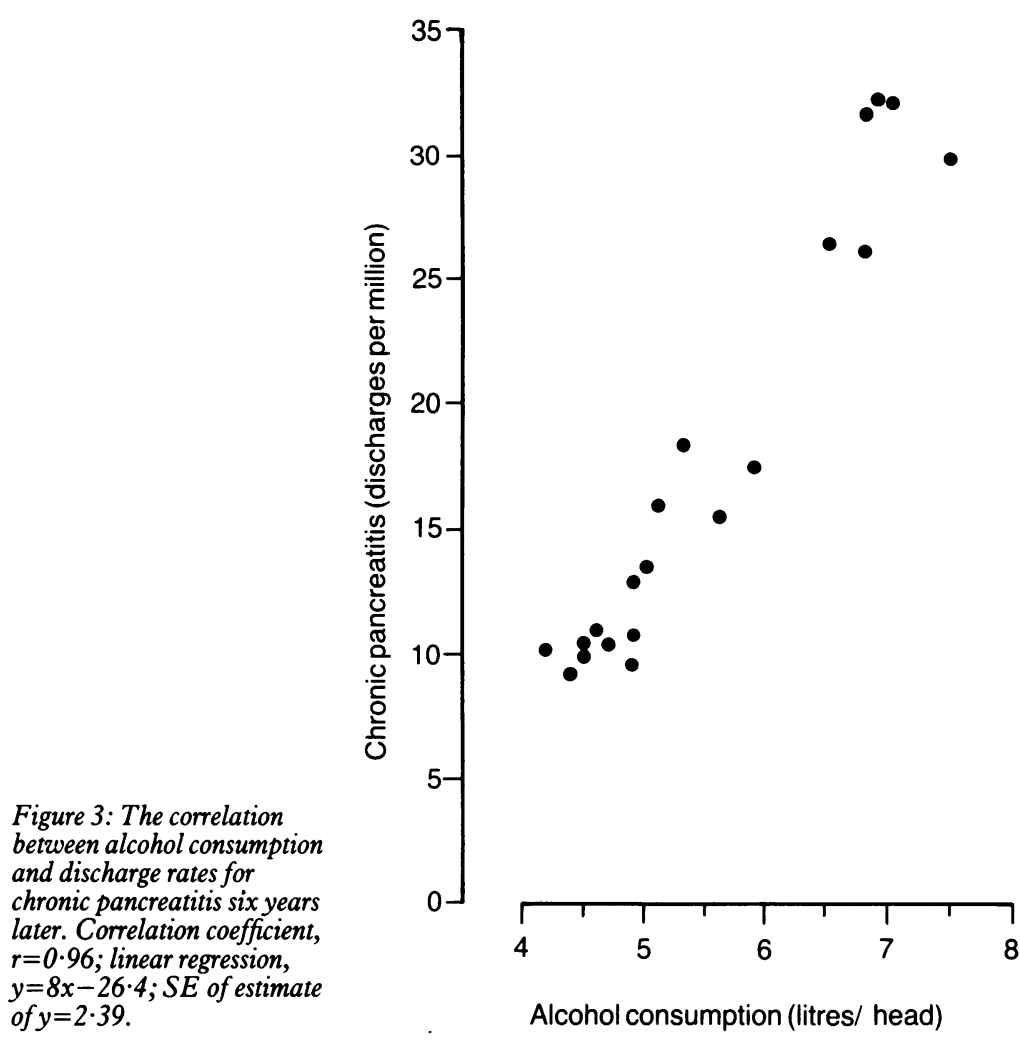

chronic pancreatitis in relation to these figures, which are derived from a $10 \%$ sample of all hospital discharges. Changes in the understanding of chronic pancreatitis, in the availability of diagnostic methods, and in the awareness of clinicians of the condition may all have contributed to the changes observed. For example, the reports of James et al from London ${ }^{7}$ and Read et al from Manchester ${ }^{8}$ may have drawn attention to this condition and led to an increased rate of diagnosis. Furthermore, during the 1970s diagnostic techniques such as ultrasound, computed tomography, and endoscopic retrograde pancreatography became widely available. These may have led to increased diagnosis of less severe cases. Nevertheless, it is unlikely that these changes could account for a threefold increase in the numbers of patients discharged from hospital with this diagnosis.

Changes in diagnostic techniques should affect equally men and women. In fact, the discharge rate in men has risen twice as much as that in women (Table) and the increase in men began several years earlier. In 1979 discharges for women exceeded 13 per million for the first time, whereas in 1974 the rate in men had already reached $20 \cdot 4$ per million. Discharge rates before 1970 were similar in both sexes at around 10 per million. These differences between men and women are strong evidence that the observed increase reflects a true increase in the disease rather than more accurate diagnosis. The higher increase in men is consistent with well known associations between male sex and other alcohol related medical conditions, alcohol related criminal behaviour, and prosecutions for drunkenness.

The observed increase in discharge rates does not equate with a threefold increase in the incidence of chronic pancreatitis because some patients may have been admitted and discharged several times. Similarly, the rate of discharges is not the same thing as prevalence. It is likely, however, that this rise in hospital discharges is indicative of a rising incidence of chronic pancreatitis, partly because of the pattern of the increase, which is not linear, and partly because of its magnitude.

The rise in the number of deaths from chronic pancreatitis also tends to confirm the observed increase in hospital discharges. There was considerable variation from year to year in the number of deaths, so no clear association with alcohol consumption or rates of hospital discharge could be determined. Nevertheless, the level of deaths has been higher in the 1980s following the rise in discharge rate in the 1970s (Fig 1).

The changes in dietary intake over the 25 years of the study are of interest, but explanations for these changes can only be speculative. It seems unlikely that meals consumed outside the home have changed to a sufficient extent to affect the observations significantly. The National Food Survey does not include sweets, chocolate, or alcohol in the estimate of total energy consumption. The figures for consumption of confectionery show some differences in sugar and chocolate based products and variable changes in total consumption over the period of study. These changes cannot account for the progressive fall in carbohydrate consumption from 1960 onwards. The calorific value of alcohol, which is now consumed in greater quantities, may have played a part in the reduction of carbohydrate consumption.

Others have examined the relation between diet and chronic pancreatitis in case-control studies. Increasing protein consumption and extremes of fat consumption are associated with chronic pancreatitis.' Two North American studies $^{39}$ reported that high alcohol consumption was associated with low fat, protein, and carbohydrate consumption. While alcohol consumption remains the major determinant for chronic pancreatitis, we have evidence that a high fat, high protein diet seems to contribute in many countries in Europe and South America. ${ }^{10}$ The interplay between all these factors is complex and our own data are too broad to enable any further insight to the contribution of diet.

Recently, attention has focused on oxidants as a possible pathogenic factor in chronic pancreatitis." Oxidants induce cytochrome P450 which may have a deleterious effect on the pancreas by the increased generation of highly reactive metabolites. Characterisation of ingested food by oxidant and antioxidant activity may be more relevant than estimation of the quantities of fat, protein, or carbohydrate consumed. ${ }^{12}$

The increase in alcohol consumption over the period under study is not surprising. The trend was noted in the $1970 \mathrm{~s}^{78}$ and until 1979 had risen rapidly. Consumption fell after this, but since 1983 the annual consumption of alcohol seems to have risen again more slowly, to approach the 1979 level. Consumption of wine and spirits has increased proportionately more than consumption of beer during this period, ${ }^{5}$ but Durbec and Sarles' showed that the quantity of alcohol 
consumed was the relevant factor, not the type of alcoholic beverage. Similarly, they found that the mean daily consumption was a better indicator of risk for pancreatitis than total lifetime alcohol consumption..$^{13}$ In other words, a heavy consumption for a short duration was a greater risk for pancreatitis than a moderate consumption over many years.

The increase in alcohol consumption in the United Kingdom was most rapid in 1970-80. This was followed five to seven years later by a rise in the number of patients with chronic pancreatitis discharged from hospital. Although there are some possible alternative explanations for this rise, which are outlined above, the correlation with the preceding figures for alcohol consumption is striking. Persuasive evidence for an association between these two observations is that both alcohol consumption, and the number of patients discharged with a diagnosis of chronic pancreatitis seem to have stabilised after the rapid rises in parallel of the $1970 \mathrm{~s}$. With alcohol consumption increasing slowly again after 1983, we await with interest the publication of hospital activity data (Korner) from 1985 onwards to determine what effect may be seen on hospital admissions for chronic pancreatitis and whether the incidence of chronic pancreatitis rises once more. We observed a latency of six years between mean annual per capita alcohol consumption and changes in chronic pancreatitis discharges. This contrasts with the finding of others ${ }^{129}$ who observed a mean duration of alcohol consumption of 17 years for men and 11 years for women before presentation with chronic pancreatitis (although the reported ranges of duration of consumption are wide: $3-30$ years). This difference is almost certainly related to the method of data collection. In the papers cited, patients with pancreatic disease were questioned on the duration of alcohol consumption before clinical presentation. In contrast, our approach correlates alcohol consumption and the recorded number of hospital discharges with chronic pancreatitis in a total population. The close correlation observed suggests that it may be possible to predict changes in hospital discharges of chronic pancreatitis after changes in national consumption of alcohol.

We have observed an increase in the rate of diagnosis of chronic pancreatitis in patients discharged from hospital. We have been unable to determine any association with changes in dietary consumption, as recorded in national statistics, but we have found a close correlation between the rate of diagnosis of chronic pancreatitis and mean per capita annual consumption of alcohol five to seven years previously.

We are grateful to Dr D Coggan, MRC Epidemiology Unit, Southampton, for helpful advice, and to Dr M J Campbell and $\mathrm{Mr}$ M A Mullee, Department of Medical Statistics and Computing, University of Southampton, who advised on and performed some University of Southampto
of the statistical analyses.

1 Durbec JP, Sarles H. Multicentre survey on the aetiology of pancreatic diseases. Digestion 1978; 18: 337-50.

2 Sarles H. An international survey on nutrition and pancreatitis. Digestion 1973; 9: 389-403.

3 Pitchumoni CS, Sonnershein M, Candido FM, Panchacharam $\mathrm{P}$, Cooperman JM. Nutrition in the pathogenesis of alcoholic pancreatitis. Am f Clin Nutr 1980; 33: 631-6.

4 Statistical Yearbook. London: The Biscuit, Cake, Chocolate and Confectionery Alliance, 1989.

5 The Brewer's Society UK Statistical Handbook. London: Brewing Publications Ltd, 1989.

6 Draper N, Smith H. Applied regression analysis. 2nd ed. New York: Wiley Interscience, 1981

7 James O, Agnew JE, Bouchier IAD. Chronic pancreatitis in England: a changing picture? BMF 1974; $2: 34-8$.

8 Read AG, Braganza JM, Howat HT. Pancreatitis. A retrospective study. Gut 1976; 17: 945-52.

9 Mezey E, Kolman CJ, Mae Diehal A, Mitchell MC, Herlong HF. Alcohol and dietary intake in the development of chronic pancreatitis and liver diseases. Am f Clin Nutr 1988; 48: $148-51$.

10 Sarles H, Johnson CD, Saunière J-F. Pancreatitis. Paris: Arnette (in press)

11 Acheson DWK, Rose P, Houston JB, Braganza JM. Induction of cytochrome $\mathbf{P 4 5 0}$ in pancreatic disease: consequence, coincidence or cause? Clin Chim Acta 1986; 153: 73-84.

12 Rose P, Fraine E, Hunt LP, Acheson DW, Braganza JN. Dietary antioxidants and chronic pancreatitis. Hum Nutr Clin Nutr 1986; 40: 151-64.

13 Durbec JP, Bidart JM, Sarles H. Interactions between alcohol and foodstuffs. Epidemiological aspects. Colloques de l'Inserm 1980; 95: 33-52. 